Quim. Nova, Vol. 35, No. 6, 1264-1270, 2012

\title{
ANÁLISE VIBRACIONAL DE COMPOSTOS DE COORDENAÇÃO DE NIQUEL(II): UMA ABORDAGEM AO ENSINO DOS GRUPOS PONTUAIS
}

\author{
Sergio Kogikoski Jr., Juliana dos Santos de Souza, Paula Homem-de-Mello, Herculano Martinho e Wendel Andrade Alves* \\ Centro de Ciências Naturais e Humanas, Universidade Federal do ABC, Rua Santa Adélia, 166, 09210-170 Santo André - SP, Brasil \\ Rômulo Augusto Ando
}

Instituto de Química, Universidade de São Paulo, CP 26077, 05513-970 São Paulo - SP, Brasil

Recebido em 8/9/11; aceito em 22/12/11; publicado na web em 23/3/12

\begin{abstract}
VIBRATIONAL ANALYSIS OF COORDINATION COMPOUNDS OF NICKEL (II): AN APPROACH TO THE TEACHING OF POINT GROUPS. This paper presents an IR and Raman experiment executed during the teaching of the course "Chemical Bonds" for undergraduated students of Science and Technology and Chemistry at the Federal University of ABC, in order to facilitate and encourage the teaching and learning of group theory. Some key aspects of this theory are also outlined. We believe that student learning was more significant with the introduction of this experiment, because there was an increase in the discussions level and in the performance during evaluations. This work also proposes a multidisciplinary approach to include the use of quantum chemistry tools.
\end{abstract}

Keywords: group theory; didactic experiment; IR and Raman spectroscopy.

\section{INTRODUÇÃO}

A teoria de grupos é um ramo da matemática que se dedica a estudar estruturas algébricas chamadas de grupos. Essa teoria foi proposta inicialmente por Évariste Galois (1811-32), no início do século XIX, para resolver equações de graus superiores a quatro. De fato, a de grupos foi revolucionária e propiciou o surgimento de uma nova álgebra. ${ }^{1}$ Outros pesquisadores que contribuíram para o desenvolvimento inicial da teoria de grupos foram Augustin L. Cauchy (1789-1857), Arthur Cayley (1821-95), Camille M. E. Jordan (1838-1922), Marius S. Lie (1842-99) e Ludwig Sylow (1832-1918). Na área de Química, a parte mais aplicada da teoria de grupos é a teoria da representação, desenvolvida por George F. Frobenius (1849-1917). ${ }^{2}$

Uma das primeiras aplicações da teoria de grupos foi na análise de dados cristalográficos, proposta por Artur Schoenflies, em 1923. Hermann Weyl (1885-1955) e Eugene P. Wigner (1902-1995) desenvolveram a relação entre teoria de grupos e mecânica quântica. ${ }^{2}$

Pode-se afirmar que a teoria de grupos é uma abstração das propriedades de simetria de um sistema. O conjunto das transformações sobre as quais um dado objeto é invariante é definido como grupo. Estas transformações ou operações de simetria que estão associadas a propriedades invariantes do sistema são importantes na previsão de propriedades físicas (como polaridade e quiralidade), no estabelecimento de regras de seleção em espectroscopia, na construção de orbitais moleculares e, mesmo, na previsão de reatividade e de mecanismos, sendo uma ferramenta adequada para o estudo de sistemas inorgânicos. ${ }^{3}$

Uma operação de simetria consiste em mover um corpo de tal maneira que a posição final é indistinguível da inicial. As operações de simetria típicas são as rotações, reflexões e inversões. ${ }^{3}$

Para cada operação de simetria há um elemento de simetria correspondente, que é um ponto, uma linha (eixo de simetria) ou um plano, em relação ao qual se faz a operação de simetria, tais como:

1. planos de simetria e reflexões $(\sigma)$ - um plano de simetria de um corpo deve passar através do mesmo, de maneira que ao se aplicar uma operação de reflexão a todos os seus componentes

\footnotetext{
*e-mail: wendel.alves@ufabc.edu.br
}

(átomos no caso de uma molécula) se chegue a uma configuração equivalente à inicial;

2. centro de inversão (i) - se, mediante transformação das coordenadas que leva $(\mathrm{x}, \mathrm{y}, \mathrm{z})$ para $(-\mathrm{x},-\mathrm{y},-\mathrm{z})$ de todos os átomos de uma molécula, esta fica numa configuração equivalente à original, diz que há um centro de inversão. Exemplos: $\mathrm{CO}_{2}, \mathrm{SF}_{6}, \mathrm{~N}_{2}$;

3. eixos próprios e rotações próprias $\left(\mathrm{C}_{\mathrm{n}}\right)$ : moléculas que apresentam um eixo $C_{n}$ podem girar em torno desse eixo sem que sua posição se modifique, ou seja, após a rotação a molécula não pode ser diferenciada da molécula antes da rotação; essa rotação é caracterizada pelo ângulo de rotação $\theta=2 \pi / n$, onde $n$ é a ordem da rotação e expressa a fração do giro completo;

4. eixos impróprios e rotações impróprias $\left(\mathrm{S}_{\mathrm{n}}\right)$ - uma rotação imprópria é uma operação de simetria que ocorre em duas etapas: uma rotação e, em seguida, uma reflexão num plano perpendicular ao eixo de rotação;

5. identidade (E) - é o elemento de simetria que não altera a posição da molécula, o que significa a rotação da mesma em $2 \varpi$.

Todas as possíveis operações de simetria no espaço são agrupadas em 32 conjuntos, chamados de grupos pontuais. ${ }^{4}$ Átomos individuais e moléculas têm simetrias espaciais caracterizadas por seu grupo pontual, que consiste no conjunto de rotações e reflexões que deixa o átomo ou molécula invariante. Os arranjos atômicos em uma rede cristalina regular são caracterizados por um grupo espacial que contém translações em adição às reflexões e rotações. ${ }^{3,5}$

Cada molécula pode ser atribuída a um grupo de ponto, considerando-se os elementos de simetria que possui. Os grupos de ponto podem ser classificados, empregando-se a notação de Schönflies, em: grupos de rotações simples $\left(\mathrm{C}_{\mathrm{n}}, \mathrm{C}_{\mathrm{nv}}, \mathrm{C}_{\mathrm{nh}}, \mathrm{S}_{\mathrm{n}}, \mathrm{D}_{\mathrm{n}}, \mathrm{D}_{\mathrm{nd}}, \mathrm{D}_{\mathrm{nh}}\right)$; grupos de alta simetria $\left(\mathrm{T}, \mathrm{T}_{\mathrm{d}}, \mathrm{T}_{\mathrm{h}}, \mathrm{O}, \mathrm{O}_{\mathrm{h}}\right)$ e grupo das moléculas lineares $\left(\mathrm{C}_{\infty \mathrm{v}} \text { e } \mathrm{D}_{\infty \mathrm{h}}\right)^{3}{ }^{3}$

Conhecendo-se o grupo ponto presente na molécula é possível conhecer os elementos de simetria associados a ela e, com isso, relacioná-los com o comportamento de simetria dos graus de liberdade. Esses graus de liberdade podem, então, ser representados por vetores cujas características de simetria podem ser elucidadas com auxílio da teoria de grupos. Esses vetores, ou coordenadas normais, serão utilizados para a obtenção da tabela de caracteres do grupo de ponto sendo estudado e podem ser divididos em vetores translacionais, rotacionais e vibracionais. 
Os vetores translacionais podem ser representados por um vetor $\mathrm{T}$, que pode ser decomposto em suas três componentes ortogonais $\mathrm{T}_{\mathrm{x}}, \mathrm{T}_{\mathrm{y}}$ e $\mathrm{T}_{\mathrm{z}}$. Movimentos translacionais são relacionados com a movimentação da molécula como um todo, ou seja, um movimento do centro de massa da molécula. Sobre esses vetores podem-se aplicar as operações de simetria do grupo de ponto da molécula estudada e, com isso, montar uma matriz que represente a aplicação dessa operação de simetria no grupo de ponto. ${ }^{5}$ Como, por exemplo, a aplicação das operações de simetria sobre uma molécula com grupo de ponto $\mathrm{C}_{2 \mathrm{v}}$ (como a água, por exemplo) gera uma mudança no sentido dos vetores translacionais (Figura 1) e por serem vetores podem gerar representações matriciais (Equações 1-4).

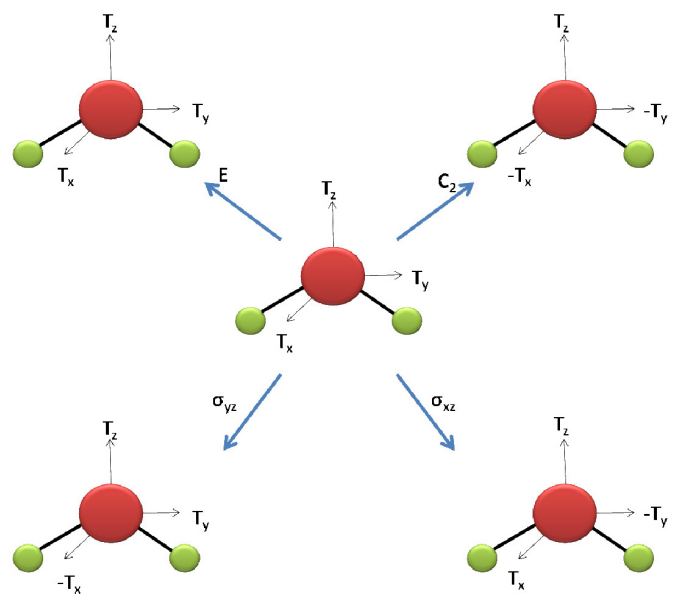

Figura 1. Aplicação das operações de simetria sobre os vetores translacionais em uma molécula com simetria $C_{2 v}$

$$
\begin{aligned}
& \begin{array}{c}
T_{x}^{\prime}=(1) \cdot T_{x} \\
T_{y}^{\prime}=(1) \cdot T_{y} \\
T_{z}^{\prime}=(1) \cdot T_{z}
\end{array} \rightarrow\left(\begin{array}{l}
T_{x}^{\prime} \\
T_{y}^{\prime} \\
T_{z}^{\prime}
\end{array}\right)=E \times\left(\begin{array}{l}
T_{x} \\
T_{y} \\
T_{z}
\end{array}\right)=\left(\begin{array}{lll}
1 & 0 & 0 \\
0 & 1 & 0 \\
0 & 0 & 1
\end{array}\right)\left(\begin{array}{l}
T_{x} \\
T_{y} \\
T_{z}
\end{array}\right) \\
& \begin{array}{c}
T_{x}^{\prime}=(-1) \cdot T_{x} \\
T_{y}^{\prime}=(-1) \cdot T_{y} \\
T_{z}^{\prime}=(1) \cdot T_{z}
\end{array} \rightarrow\left(\begin{array}{c}
T_{x}^{\prime} \\
T_{y}^{\prime} \\
T_{z}^{\prime}
\end{array}\right)=C_{2} \times\left(\begin{array}{l}
T_{x} \\
T_{y} \\
T_{z}
\end{array}\right)=\left(\begin{array}{ccc}
-1 & 0 & 0 \\
0 & -1 & 0 \\
0 & 0 & 1
\end{array}\right)\left(\begin{array}{l}
T_{x} \\
T_{y} \\
T_{z}
\end{array}\right) \\
& \begin{array}{l}
T_{x}^{\prime}=(1) \cdot T_{x} \\
T_{y}^{\prime}=(-1) \cdot T_{y}
\end{array} \rightarrow\left(\begin{array}{l}
T_{x}^{\prime} \\
T_{y}^{\prime} \\
T_{z}^{\prime}
\end{array}\right)=\sigma_{x z} \times\left(\begin{array}{l}
T_{x} \\
T_{y} \\
T_{z}
\end{array}\right)=\left(\begin{array}{ccc}
1 & 0 & 0 \\
0 & -1 & 0 \\
0 & 0 & 1
\end{array}\right)\left(\begin{array}{l}
T_{x} \\
T_{y} \\
T_{z}
\end{array}\right) \\
& T_{z}^{\prime}=(1) \cdot T_{z} \quad\left(\begin{array}{l}
T_{z}^{\prime}
\end{array}\right)=\sigma_{x z} \times\left(\begin{array}{l}
T_{2} \\
T_{z}
\end{array}\right)=\left(\begin{array}{lll}
0 & -1 & 0 \\
0 & 0 & 1
\end{array}\right)\left(\begin{array}{l}
T_{2} \\
T_{z}
\end{array}\right) \\
& \begin{array}{c}
T_{x}^{\prime}=(-1) \cdot T_{x} \\
\sigma_{y z}^{\prime}=(1) \cdot T_{y} \\
T_{z}^{\prime}=(1) \cdot T_{z}
\end{array} \rightarrow\left(\begin{array}{c}
T_{x}^{\prime} \\
T_{y}^{\prime} \\
T_{z}^{\prime}
\end{array}\right)=\sigma_{y z} \times\left(\begin{array}{c}
T_{x} \\
T_{y} \\
T_{z}
\end{array}\right)=\left(\begin{array}{ccc}
-1 & 0 & 0 \\
0 & 1 & 0 \\
0 & 0 & 1
\end{array}\right)\left(\begin{array}{c}
T_{x} \\
T_{y} \\
T_{z}
\end{array}\right)
\end{aligned}
$$

Essa representação matricial introduz a definição de caractere redutível e irredutível, que dependerá da escolha da base para a representação; por exemplo, se for usada como base as coordenadas cartesianas de deslocamento ter-se-á um caráter redutível e se for utilizada a base formada pelos vetores translacionais obtém-se um caráter irredutível. Uma propriedade importante desta representação é que os caracteres das matrizes formam representações do grupo de ponto, o que facilita o problema, sendo possível trabalhar com números ao invés de matrizes. ${ }^{5}$

No caso dos movimentos rotacionais, os vetores podem ser representados por um vetor $\mathrm{R}$ que corresponde ao momento rotacional (angular), é perpendicular ao plano de rotação da molécula $(\omega)$ e seu sentido é dado pela "regra da mão direita". Quando ocorre a inversão da direção do sentido de rotação, por consequência ocorre também a mudança do sinal do vetor rotacional e, de modo análogo ao vetor translacional, esse vetor também pode ser decomposto em três componentes ortogonais $\mathrm{R}_{\mathrm{x}}, \mathrm{R}_{\mathrm{y}}$ e $\mathrm{R}_{\mathrm{z}}$, mas nesse caso ao invés de aplicar as operações de simetria ao vetor, a operação é aplicada ao movimento rotacional da molécula. ${ }^{5}$ Considerando novamente o grupo de ponto $\mathrm{C}_{2 \mathrm{v}}$, as operações de simetria podem ser aplicadas ao sistema (Figura 2) e as matrizes construídas após a aplicação das operações de simetria são as dadas pelas Equações 5-8.

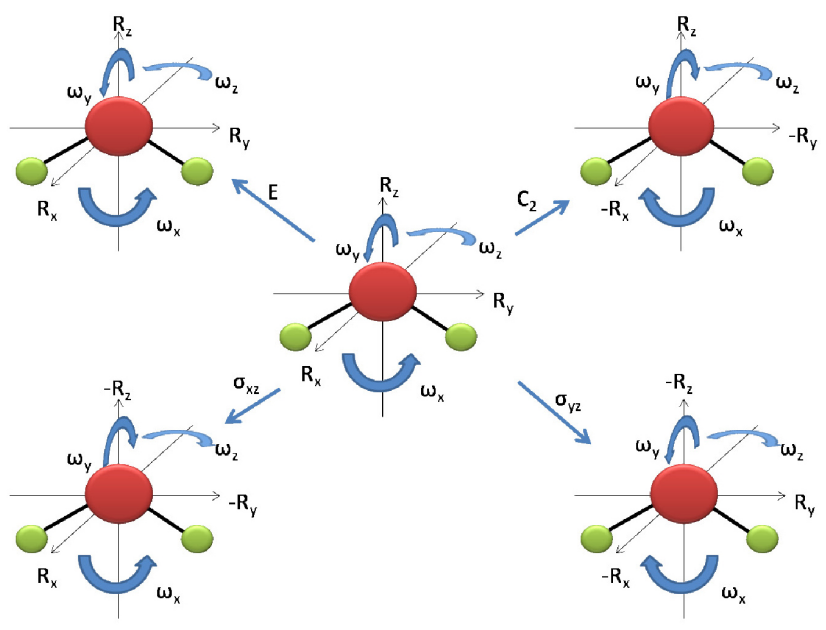

Figura 2. Aplicação das operações de simetria sobre os movimentos rotacionais em uma molécula com simetria $C_{2 v}$

$$
\begin{aligned}
& E \rightarrow \begin{array}{l}
R_{x}^{\prime}=(1) \cdot R_{x} \\
R_{y}^{\prime}=(1) \cdot R_{y} \\
R_{z}^{\prime}=(1) \cdot R_{z}
\end{array} \rightarrow\left(\begin{array}{l}
R_{x}^{\prime} \\
R_{y}^{\prime} \\
R_{z}^{\prime}
\end{array}\right)=E \times\left(\begin{array}{l}
R_{x} \\
R_{y} \\
R_{z}
\end{array}\right)=\left(\begin{array}{lll}
1 & 0 & 0 \\
0 & 1 & 0 \\
0 & 0 & 1
\end{array}\right)\left(\begin{array}{l}
R_{x} \\
R_{y} \\
R_{z}
\end{array}\right)
\end{aligned}
$$

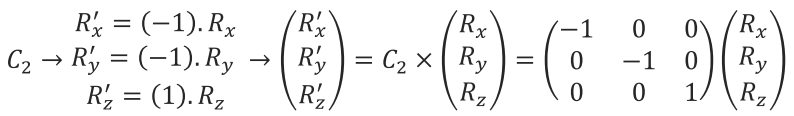

$$
\begin{aligned}
& \sigma_{x z} \rightarrow \begin{array}{l}
R_{x}^{\prime}=(-1) \cdot R_{x} \\
R_{y}^{\prime}=(1) \cdot R_{y} \\
R_{z}^{\prime}=(-1) \cdot R_{z}
\end{array} \rightarrow\left(\begin{array}{l}
R_{x}^{\prime} \\
R_{y}^{\prime} \\
R_{z}^{\prime}
\end{array}\right)=\sigma_{x z} \times\left(\begin{array}{l}
R_{x} \\
R_{y} \\
R_{z}
\end{array}\right)=\left(\begin{array}{ccc}
-1 & 0 & 0 \\
0 & 1 & 0 \\
0 & 0 & -1
\end{array}\right)\left(\begin{array}{l}
R_{x} \\
R_{y} \\
R_{z}
\end{array}\right)(7)
\end{aligned}
$$

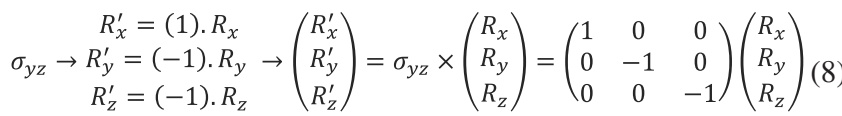

De forma análoga aos vetores translacionais, foram encontrados os caracteres reduzíveis e irredutíveis para as componentes rotacionais do grupo de ponto. Por fim, pode-se utilizar como base os vetores de deslocamento, que representam os movimentos dos átomos na direção das amplitudes vibracionais. Esses vetores serão, então, relacionados com os modos normais de vibração do grupo de ponto estudado. Lembrando que a soma vetorial dos deslocamentos deve ser igual a zero, porque o centro de massa da molécula não pode sofrer deslocamento durante o movimento vibracional. Conhecendo-se os modos vibracionais do grupo de ponto podem-se aplicar as operações de simetria, de forma a obter a sua matriz correspondente. ${ }^{5}$ No caso do grupo de ponto $\mathrm{C}_{2 \mathrm{v}}$ existem 3 modos vibracionais $v_{1}, v_{2}$ e $v_{3}$ (Figura 3A) e a aplicação das operações de simetria em um dos modos vibracionais encontra-se na Figura 3B; a partir disso, as matrizes que representam as operações de simetria relacionadas às vibrações são obtidas (Equações 9-12).

Com todos os caracteres irredutíveis obtidos para cada uma das representações vetoriais, é possível se obter uma tabela que relaciona o vetor estudado e seus caracteres irredutíveis para cada uma das operações de simetria, Tabela 1. 

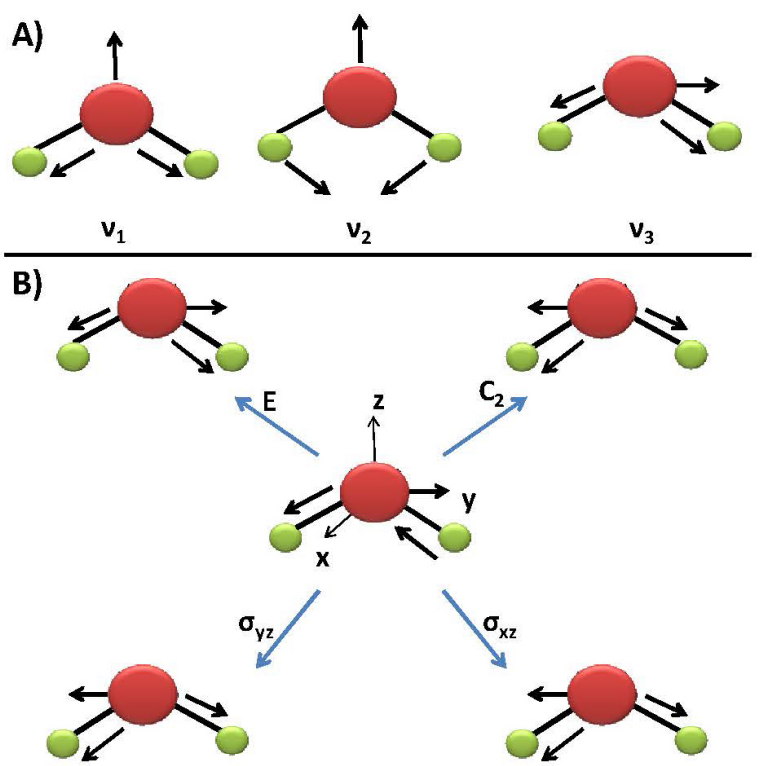

Figura 3. (A) Modos vibracionais de uma molécula com simetria $C_{2 v}$ (B) Aplicação das operações de simetria sobre um dos modos vibracionais em uma molécula com simetria $C_{2 v}$

$\begin{aligned} v_{1}^{\prime} & =(1) \cdot v_{1} \\ E \rightarrow v_{2}^{\prime} & =(1) \cdot v_{2} \\ v_{3}^{\prime} & =(1) \cdot v_{3}\end{aligned} \rightarrow\left(\begin{array}{l}v_{1}^{\prime} \\ v_{2}^{\prime} \\ v_{3}^{\prime}\end{array}\right)=E \times\left(\begin{array}{l}v_{1} \\ v_{2} \\ v_{3}\end{array}\right)=\left(\begin{array}{lll}1 & 0 & 0 \\ 0 & 1 & 0 \\ 0 & 0 & 1\end{array}\right)\left(\begin{array}{l}v_{1} \\ v_{2} \\ v_{3}\end{array}\right)$

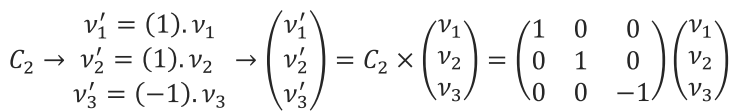

$\sigma_{x z} \rightarrow \begin{gathered}v_{1}^{\prime}=(1) \cdot v_{1} \\ v_{2}^{\prime}=(1) \cdot v_{2} \\ v_{3}^{\prime}=(-1) \cdot v_{3}\end{gathered} \rightarrow\left(\begin{array}{l}v_{1}^{\prime} \\ v_{2}^{\prime} \\ v_{3}^{\prime}\end{array}\right)=\sigma_{x z} \times\left(\begin{array}{l}v_{1} \\ v_{2} \\ v_{3}\end{array}\right)=\left(\begin{array}{ccc}1 & 0 & 0 \\ 0 & 1 & 0 \\ 0 & 0 & -1\end{array}\right)\left(\begin{array}{l}v_{1} \\ v_{2} \\ v_{3}\end{array}\right)$

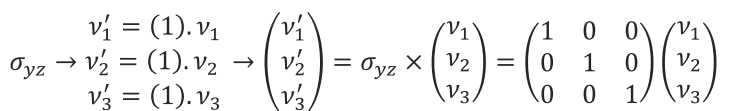

Tabela 1. Caracteres irredutíveis de T, R e V para uma molécula com simetria $\mathrm{C}_{2 \mathrm{v}}$

\begin{tabular}{cccccc}
\hline $\mathrm{C}_{2 \mathrm{v}}$ & $\mathrm{E}$ & $\mathrm{C}_{2}$ & $\sigma_{\mathrm{xz}}$ & $\sigma_{\mathrm{yz}}$ & \\
\hline $\mathrm{T}_{\mathrm{x}}$ & 1 & -1 & 1 & -1 & (a) \\
$\mathrm{T}_{\mathrm{y}}$ & 1 & -1 & -1 & 1 & (b) \\
$\mathrm{T}_{\mathrm{z}}$ & 1 & 1 & 1 & 1 & (c) \\
$\mathrm{R}_{\mathrm{x}}$ & 1 & -1 & -1 & 1 & (b) \\
$\mathrm{R}_{\mathrm{y}}$ & 1 & -1 & 1 & -1 & (a) \\
$\mathrm{R}_{\mathrm{z}}$ & 1 & 1 & -1 & -1 & (d) \\
$\mathrm{V}_{1}$ & 1 & 1 & 1 & 1 & (c) \\
$\mathrm{V}_{2}$ & 1 & 1 & 1 & 1 & (c) \\
$\mathrm{V}_{3}$ & 1 & -1 & -1 & 1 & (b) \\
\hline
\end{tabular}

Com isso a tabela de caracteres (Tabela 2) é, então, obtida agrupando-se as espécies com caracteres irredutíveis iguais e, com isso, é feita a atribuição dos símbolos de Mulliken, que é uma informação do grau de degeneração da matriz irredutível; o índice exprime o comportamento da simetria em relação a operações de simetria $\left(C_{n}, i, \sigma_{h}\right.$ e $\left.\sigma_{v}\right)$.
Tabela 2. Tabela de caracteres para o grupo pontual $\mathrm{C}_{2 \mathrm{v}}$

\begin{tabular}{ccccccc}
\hline $\mathrm{C}_{2 \mathrm{v}}$ & $\mathrm{E}$ & $\mathrm{C}_{2}$ & $\sigma_{\mathrm{xz}}$ & $\sigma_{\mathrm{yz}}$ & \multicolumn{2}{c}{ Graus de liberdade } \\
\hline $\mathrm{A}_{1}$ & 1 & 1 & 1 & 1 & $\mathrm{~T}_{\mathrm{z}}$ & $\mathrm{V}_{1}, \mathrm{~V}_{2}$ \\
$\mathrm{~A}_{2}$ & 1 & 1 & -1 & -1 & $\mathrm{R}_{\mathrm{z}}$ & \\
$\mathrm{B}_{1}$ & 1 & -1 & 1 & -1 & $\mathrm{~T}_{\mathrm{x}}, \mathrm{R}_{\mathrm{y}}$ & \\
$\mathrm{B}_{2}$ & 1 & -1 & -1 & 1 & $\mathrm{~T}_{\mathrm{y}}, \mathrm{R}_{\mathrm{x}}$ & $\mathrm{V}_{3}$ \\
\hline
\end{tabular}

As tabelas de caracteres mostram informações a respeito das espécies analisadas: a primeira coluna mostra o grupo de ponto da molécula e os símbolos de Mulliken associados à simetria, as próximas colunas apresentam as operações de simetria realizadas e o traço irredutível da matriz que representa essa operação. A penúltima coluna mostra quais simetrias apresentam vetores translacionais e rotacionais, que são indicados pelas funções $R_{x}, R_{y}$ ou $R_{z}$ para rotação e $T_{x}, T_{y}$ ou $T_{z}$ para translação. A última coluna mostra os vetores vibracionais do sistema, tanto os que são ativos no IR quanto os que são ativos no Raman. ${ }^{3,6-8}$

Uma das aplicações dos conceitos da teoria de grupos está na obtenção dos possíveis modos vibracionais de moléculas ou sólidos e as correspondentes regras de seleção em espectroscopia vibracional (absorção no infravermelho e espalhamento no Raman). As grandezas físicas fundamentais a serem consideradas, neste caso, são o vetor momento de dipolo elétrico, $\vec{\mu}$, e o tensor de polarizabilidade elétrica, $\vec{\alpha}$, que mensura a tendência relativa de distorção da nuvem eletrônica sob a ação de um campo elétrico externo, $\vec{E}$. As operações de simetria que deixam o arranjo atômico invariante no espaço, quando representadas em forma algébrica abstrata, também se manifestam nestas grandezas. ${ }^{6-9}$

Na espectroscopia de infravermelho, a probabilidade de transição da molécula de um estado vibracional inicial $\left(\Psi_{i}\right)$ para um estado final $\left(\Psi_{f}\right)$ depende do operador momento de dipolo elétrico, de componentes $\mu_{\mathrm{x}}, \mu_{\mathrm{y}}$ e $\mu_{\mathrm{z}}$. Diz-se, então, que o operador transforma como as coordenadas x, y e z (Equação 13).

$$
M_{x f i}=-m \int \Psi_{f}^{*} x \Psi_{i} d \tau
$$

As funções de onda vibracionais têm a simetria dos polinômios de Hermite, ou seja, a simetria de uma coordenada. Os operadores dipolo elétrico transformam como produtos cartesianos $\mathrm{x}, \mathrm{y}, \mathrm{z}$. Se a espécie de simetria de um modo normal de vibração tem a espécie de simetria de uma coordenada $(\mathrm{x}, \mathrm{y}, \mathrm{z})$ esta vibração será ativa no infravermelho. Portanto, para saber se um dado modo normal é ativo no infravermelho basta observar na tabela de caracteres se sua espécie de simetria tem alguma coordenada como função base. ${ }^{6,8}$

Por outro lado, o efeito Raman depende de um momento de dipolo induzido na molécula devido ao campo eletromagnético da radiação incidente. $\mathrm{O}$ momento de dipolo induzido é proporcional à polarizabilidade $\alpha$ da molécula. A polarizabilidade $\alpha$ é um tensor, ao invés de ser um vetor, como em moléculas de alta simetria (tetraédricas e octaédricas, por exemplo) o campo elétrico externo irá gerar um momento de dipolo induzido com direção igual à do campo elétrico, independente da orientação da molécula. Nesse tipo de molécula quando o vetor campo elétrico é decomposto, a contribuição para cada vetor momento de dipolo induzido fica $\overrightarrow{\mu_{x}}=\alpha \overrightarrow{E_{x}}, \overrightarrow{\mu_{y}}=\alpha \overrightarrow{E_{y}} e \overrightarrow{\mu_{z}}=\alpha \overrightarrow{E_{z}}$, nesse caso o valor de $\alpha$ é o mesmo em qualquer direção e a molécula é chamada de isotrópica. Para os casos onde o momento de dipolo induzido pode não ser paralelo ao campo elétrico, essas moléculas são chamadas de anisotrópicas, já que quando um componente campo elétrico $\mathrm{E}$ induz um momento de dipolo que possui um componente em determinada direção, mas também pode induzir um momento de dipolo nas outras componentes, faz com que a radiação espalhada 
contenha ondas perpendiculares entre si. ${ }^{6,8,10}$ Para um caso geral a seguinte matriz pode ser utilizada (Equação 14):

$\left(\begin{array}{l}\mu_{x} \\ \mu_{y} \\ \mu_{z}\end{array}\right)=\left(\begin{array}{lll}\alpha_{x x} & \alpha_{x y} & \alpha_{x z} \\ \alpha_{y x} & \alpha_{y y} & \alpha_{y z} \\ \alpha_{z x} & \alpha_{z y} & \alpha_{z z}\end{array}\right)\left(\begin{array}{l}E_{x} \\ E_{y} \\ E_{z}\end{array}\right)=\left(\begin{array}{ccc}x^{2} & x y & x z \\ x y & y^{2} & y z \\ x z & y z & z^{2}\end{array}\right)\left(\begin{array}{l}E_{x} \\ E_{y} \\ E_{z}\end{array}\right)$

Com isso tem-se que a vibração ativa no Raman deve mudar o momento de dipolo em pelo menos uma das direções mostradas pelo tensor polarizabilidade.

Os cálculos para a obtenção das representações vibracionais são extremamente trabalhosos, porém são importantes por mostrarem a contribuição de cada uma das operações de simetria para o modelo da vibração molecular. Primeiramente, são obtidos todos os graus de liberdade da molécula e sua representação pela simbologia de Mulliken e, então, são retirados os modos translacionais e rotacionais, obtendo-se assim todos os modos ativos no IR e Raman. Retirando-se as funções que representam os modos ativos no Raman, obtém-se a representação irredutível das vibrações ativas no IR..$^{5,11}$ Uma explicação detalhada sobre esses cálculos é encontrada no material suplementar.

Os modos vibracionais que são ativos para cada um dos grupos de ponto que serão estudados são: para molécula $\mathrm{C}_{3 \mathrm{v}}$ são ativos no infravermelho os modos $\mathrm{A}_{1}$ e $\mathrm{E}$, e são ativos no Raman os modos $\mathrm{A}_{1}$ e E; para as moléculas com simetria $\mathrm{T}_{\mathrm{d}}$, o modo vibracional $\mathrm{T}_{2}$ é ativo no infravermelho e são ativos no Raman os modos vibracionais $\mathrm{A}_{1}$, E e $\mathrm{T}_{2}$; para as moléculas com simetria $\mathrm{O}_{\mathrm{h}}$, o modo vibracional ativo no infravermelho é o $\mathrm{T}_{1 \mathrm{u}}$ e os ativos no Raman são $\mathrm{A}_{1 \mathrm{~g}}, \mathrm{E}_{\mathrm{g}}$ e $\mathrm{T}_{2 \mathrm{~g}}$ (Figura 4).

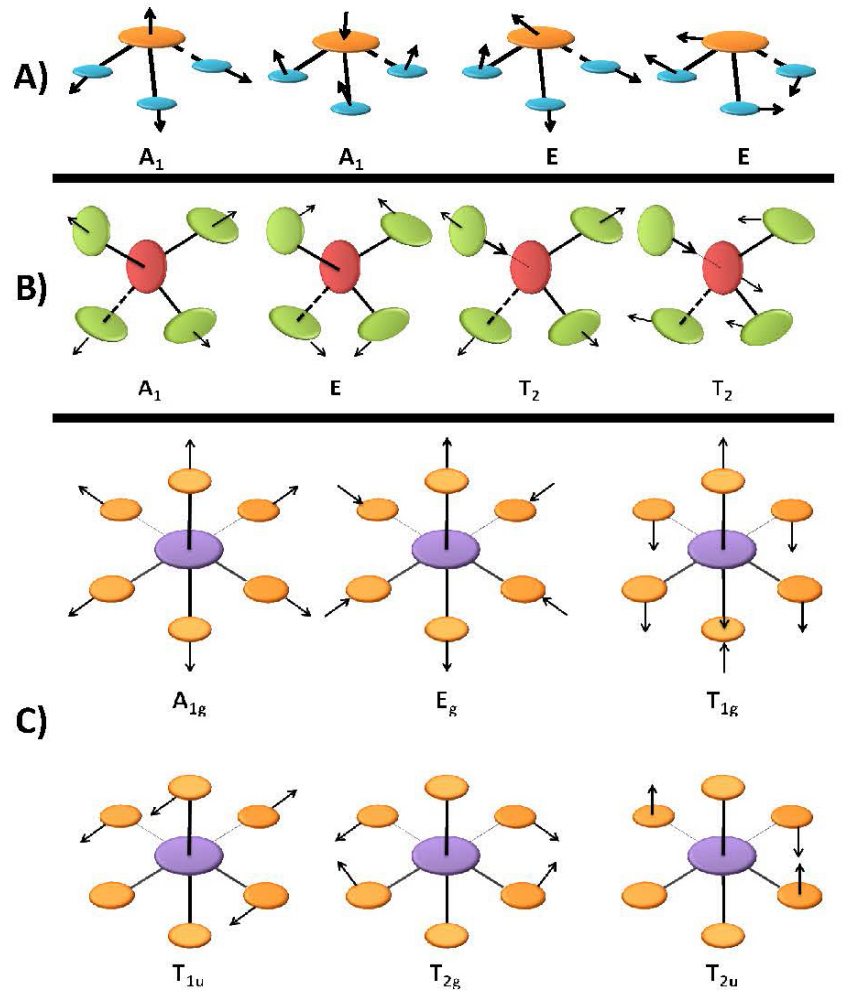

Figura 4. Modos normais de vibração dos grupos de ponto (A) $C_{3 v}$ (B) $T_{d}$ $e(C) O_{h}$

Já foram publicados trabalhos nessa área para incitar o estudo da teoria de grupos em cursos de graduação, ${ }^{12}$ outros apenas abordaram alguns fundamentos das espectroscopias (atômica e molecular). ${ }^{10,13}$ Dada a importância da teoria de grupos para a espectroscopia, neste trabalho é proposta a realização de um experimento com o intuito de facilitar o ensino e a aprendizagem de alguns conceitos envolvidos nessa área. Este experimento foi utilizado na disciplina Ligações Químicas, para os Bacharelados em Ciência e Tecnologia e em Química Fundamental da Universidade Federal do ABC (UFABC). A disciplina tem carga horária de $48 \mathrm{~h}$ e a ementa prevê o estudo das principais características da teoria de grupos, bem como o tratamento sistemático das ligações químicas e suas aplicações em espectroscopia vibracional e eletrônica.

A teoria de grupos é tradicionalmente abordada em disciplinas de Química Inorgânica, mas em alguns cursos de graduação em Química este tema é negligenciado. Acreditamos que a presença de uma proposta experimental pode ajudar no ensino e, principalmente, na compreensão dos conceitos abstratos envolvidos neste assunto.

$\mathrm{O}$ espectro eletrônico de um dos complexos do experimento aqui proposto foi utilizado por Gushikem ${ }^{14}$ para introduzir a teoria do campo cristalino no curso de graduação, em que é possível notar uma simples correlação entre a estrutura eletrônica do composto com seu espectro eletrônico e, com isso, calcular os principais parâmetros, que são o valor do desdobramento do campo cristalino (10Dq) e interação eletrônica (parâmetro de Racah), utilizando-se o diagrama de Tanabe-Sugano. ${ }^{15} \mathrm{O}$ presente trabalho é uma expansão desse estudo, abordando conceitos relacionados à espectroscopia vibracional. Neste caso, propõe-se um experimento simples no qual os alunos preparam e analisam os espectros vibracionais (infravermelho e Raman) de complexos hexacoordenados de $\left[\mathrm{Ni}\left(\mathrm{NH}_{3}\right)_{6}\right] \mathrm{X}_{2}$ $\left(\mathrm{X}=\mathrm{ClO}_{4}^{-}, \mathrm{BF}_{4}^{-}{\mathrm{e} \mathrm{PF}_{6}^{-}}^{-}\right.$.

A primeira abordagem a ser considerada é investigar apenas as propriedades de simetria de grupos moleculares específicos, que é a abordagem relevante para o entendimento das ligações químicas. São abordadas as principais características da teoria de grupos do ponto de vista químico, utilizadas para o entendimento dos espectros vibracionais, o que pode contribuir no aprendizado de conceitos fundamentais que dependem da geometria molecular. Um aprofundamento do estudo incluiria a simetria cristalina, fundamental para o estudo do estado sólido de forma mais geral.

\section{PARTE EXPERIMENTAL}

\section{Síntese dos complexos de Níquel(II)}

Os complexos $\left[\mathrm{Ni}\left(\mathrm{NH}_{3}\right)_{6}\right]\left(\mathrm{ClO}_{4}\right)_{2},\left[\mathrm{Ni}\left(\mathrm{NH}_{3}\right)_{6}\right]\left(\mathrm{BF}_{4}\right)_{2}$ e $\left[\mathrm{Ni}\left(\mathrm{NH}_{3}\right)_{6}\right]$ $\left(\mathrm{PF}_{6}\right)_{2}$ foram sintetizados de acordo com procedimento descrito na literatura. ${ }^{16}$

\section{Espectros vibracionais}

Os espectros IR foram obtidos em um espectrômetro Varian 660 IR, utilizando um acessório de ATR (reflexão total atenuada) modelo Miraclen,Pike com cristal de diamante e leitura de 4000 a $525 \mathrm{~cm}^{-1}$.

Os espectros Raman foram obtidos em um espectrômetro Renishaw InVia excitados por um laser de HeNee (Coherent) $\lambda_{0}=$ $632,8 \mathrm{~nm}$ na região de $\mathrm{n}^{\circ}$ de onda de $100-3600 \mathrm{~cm}^{-1}$ (resolução $1 \mathrm{~cm}^{-1}$ e potência $=50 \mathrm{~mW}$ ).

\section{Avaliação}

Os alunos avaliaram, de forma anônima, diversos pontos da disciplina utilizando um questionário qualitativo de quatro níveis (ótimo, bom, regular e ruim) composto de 18 questões divididas em três dimensões: planejamento e cumprimento do programa da disciplina; comunicação e uso de técnicas e recursos didáticos e, avaliação e resultados. 
O questionário é apresentado no material suplementar. Em relação especificamente ao emprego do experimento, foram levantados os seguintes pontos: correlação com conceitos apresentados na teoria, técnicas empregadas e auxílio na avaliação final.

\section{RESULTADOS E DISCUSSÃO}

Para o tratamento rigoroso dos modos vibracionais recorre-se à mecânica quântica. No caso de moléculas pequenas, algumas propriedades físicas, particularmente aquelas relacionadas com as frequências vibracionais podem ser obtidas tanto pelo método clássico, quanto pelo método quântico. Uma descrição completa sobre todo o formalismo que envolve as vibrações moleculares pode ser encontrada no livro de Sala. ${ }^{8}$

Entretanto, podem-se tratar os sistemas mais complexos decompondo-os. Cada átomo possui 3 graus de liberdade (movimentos nas direções $\mathrm{x}, \mathrm{y}$ ou $\mathrm{z}$ ) e em uma molécula com $\mathrm{N}$ átomos haverá $3 \mathrm{~N}$ graus de liberdade. Dentre os graus de liberdade possíveis, 3 são translacionais (centro de massa da molécula nas direções $\mathrm{x}, \mathrm{y}$ ou z) e 3 são rotacionais (x, y e z). Dessa forma, são obtidos 3N-6 graus de liberdade referentes aos movimentos fundamentais da molécula. ${ }^{17}$

Com base nesse conceito é possível estimar o número de modos vibracionais para as moléculas sintetizadas. Os complexos [ $\left.\mathrm{Ni}\left(\mathrm{NH}_{3}\right)_{6}\right]$ $\left(\mathrm{ClO}_{4}\right)_{2}$ e $\left[\mathrm{Ni}\left(\mathrm{NH}_{3}\right)_{6}\right]\left(\mathrm{BF}_{4}\right)_{2}$ possuem 35 átomos e, portanto, 99 modos vibracionais. Esse valor dificulta o tratamento dos dados e, por isso, considera-se que os modos são compostos de blocos independentes, de acordo com a simetria local, um referente aos modos do $\mathrm{NH}_{3}$ $\left(\mathrm{C}_{3 \mathrm{v}}\right.$, piramidal triangular), um ao contraíon e o outro às ligações entre o centro metálico e o ligante $\mathrm{NH}_{3}$. No caso do $\mathrm{NH}_{3}, 3 \mathrm{~N}-6=6$ e os contraíons $\left(\mathrm{T}_{\mathrm{d}}\right.$, geometria tetraédrica) $\mathrm{ClO}_{4}^{-}$e $\mathrm{BF}_{4}-3 \mathrm{~N}-6=9$ e, finalmente, para o contraíon $\left(\mathrm{O}_{\mathrm{h}}\right.$, geometria octaédrica) $\mathrm{PF}_{6}-3 \mathrm{~N}-6=$ 15 (Tabelas 7S-9S, material suplementar). De acordo com a teoria do campo cristalino, um complexo pode ser representado como o íon metálico e os ligantes como cargas pontuais negativas. Os complexos de níquel(II) sintetizados apresentam geometria $\mathrm{O}_{\mathrm{h}}$, onde o íon $\mathrm{Ni}(\mathrm{II})$ está ligado a seis ligantes do tipo $\mathrm{NH}_{3}$, ou seja, $3 \mathrm{~N}-6=15$.

Com base nos modelos de vibrações moleculares é possível verificar quais são os modos vibracionais ativos no IR e no Raman para o caso dos complexos estudados. No caso do ligante $\mathrm{NH}_{3}$ apenas uma das vibrações do tipo $A_{1}$ mostra variação no momento de dipolo da molécula, sendo ativo no IR, o mesmo é observado no caso de uma das vibrações do tipo E (duplamente degenerada); nos dois casos, a vibração é composta por uma deformação angular ( ) e os demais modos vibracionais são ativos no Raman. Para os contraíons tetraédricos $\mathrm{ClO}_{4}{ }^{-} \mathrm{e} \mathrm{BF}_{4}{ }^{-}$somente o modo vibracional $\mathrm{T}_{2}$ apresenta variação de $\mathrm{m}$ (ativo no IR) e os modos $\mathrm{A}_{1}$, $\mathrm{E}$ e $\mathrm{T}_{2}$ são ativos em Raman. $\mathrm{O}$ íon $\mathrm{PF}_{6}$ e o complexo analisado mostram apenas o modo vibracional $\mathrm{T}_{1 \mathrm{u}}$ ativo no IR, enquanto os demais modos, com exceção do $\mathrm{T}_{2 \mathrm{u}}$, são ativos no Raman. A vibração referente à simetria $T_{2 u}$ não é ativa tanto no IR quanto no Raman.

Dessa maneira, é possível compreender a relação entre a teoria de grupo e as bandas observadas nos espectros IR e Raman dos complexos $\left[\mathrm{Ni}\left(\mathrm{NH}_{3}\right)_{6}\right]\left(\mathrm{ClO}_{4}\right)_{2}$, Figura 5, $\left[\mathrm{Ni}\left(\mathrm{NH}_{3}\right)_{6}\right]\left(\mathrm{BF}_{4}\right)_{2}$, Figura 6 , e $\left[\mathrm{Ni}\left(\mathrm{NH}_{3}\right)_{6}\right]\left(\mathrm{PF}_{6}\right)_{2}$, Figura 7. É importante notar que um estudo semelhante foi realizado, na década de 80, por Kulczycki. ${ }^{18}$ As Tabelas 3 e 4 mostram a comparação entre os valores obtidos por Kulczycki com os obtidos neste trabalho, sendo possível observar a concordância dos resultados.

Os modos vibracionais ativos no IR são relacionados com os valores encontrados experimentalmente. No caso do $\mathrm{NH}_{3}$, duas bandas na região de 3300 a $3400 \mathrm{~cm}^{-1}$ possuem simetria $\mathrm{A}_{1}$ e E, e uma banda em cerca de $1600 \mathrm{~cm}^{-1}$ de simetria $\mathrm{E}$, atribuída à deformação angular. $\mathrm{A}$ análise dos espectros dos contraíons $\mathrm{ClO}_{4}^{-}$e $\mathrm{BF}_{4}^{-}$mostra duas

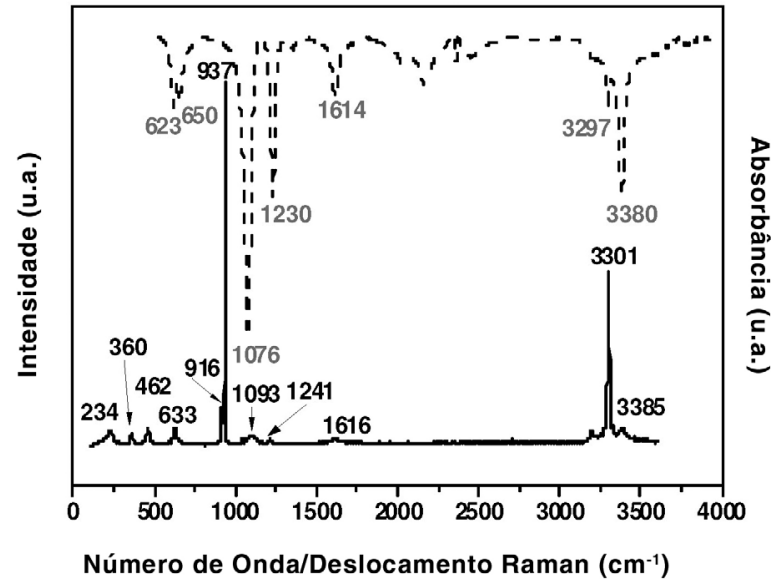

Figura 5. Espectros vibracionais, Raman (-) e infravermelho (- - -) do composto $\left[\mathrm{Ni}\left(\mathrm{NH}_{3}\right)_{6}\right]\left(\mathrm{ClO}_{4}\right)_{2}$

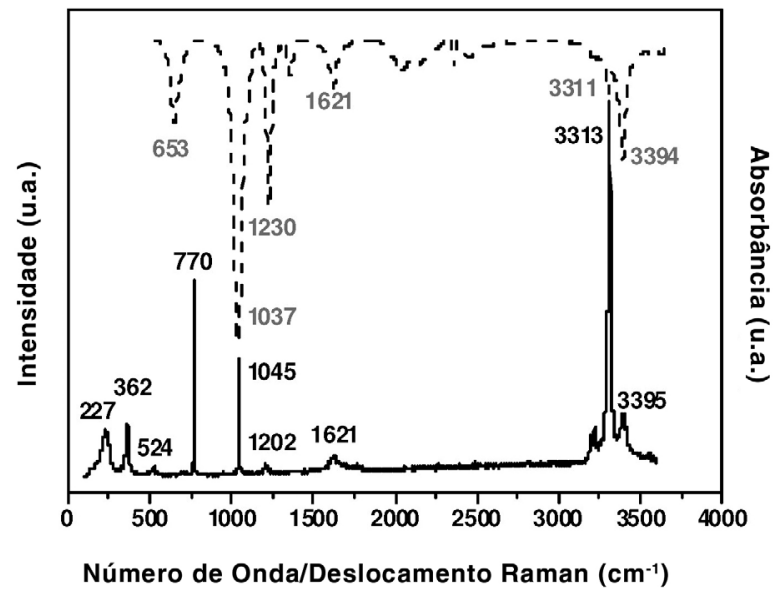

Figura 6. Espectros vibracionais, Raman (-) e infravermelho (- - -) do composto $\left[\mathrm{Ni}\left(\mathrm{NH}_{3}\right)_{6}\right]\left(\mathrm{BF}_{4}\right)_{2}$

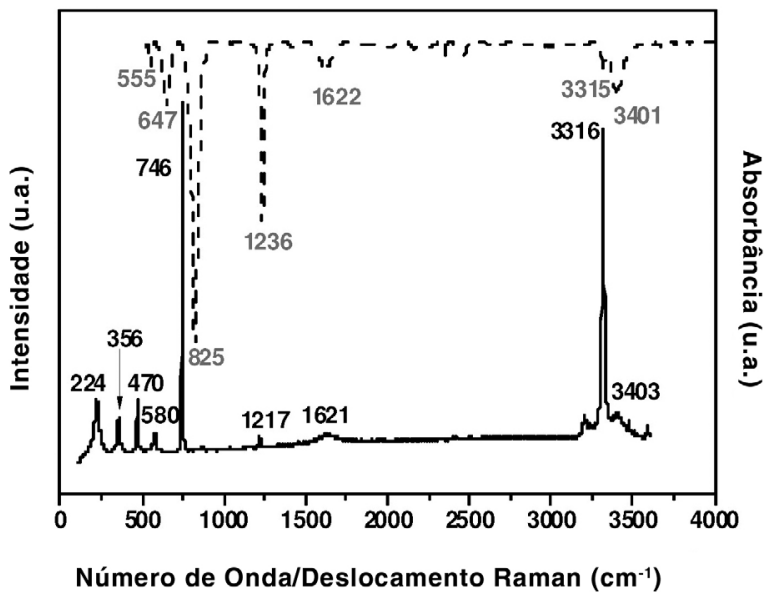

Figura 7. Espectros vibracionais, Raman (-) e infravermelho (- - -) do composto $\left[\mathrm{Ni}\left(\mathrm{NH}_{3}\right)_{6}\right]\left(\mathrm{PF}_{6}\right)_{2}$

bandas referentes aos modos vibracionais triplamente degenerados, $\mathrm{T}_{2}$, em 620 e $1080 \mathrm{~cm}^{-1}$, no caso do $\mathrm{ClO}_{4}^{-}$, entre 550 e $1040 \mathrm{~cm}^{-1}$ e no caso do $\mathrm{BF}_{4}^{-}$, também são atribuídos a deformações angulares. $\mathrm{O}$ íon $\mathrm{PF}_{6}^{-}$apresenta duas bandas, em 560 e $825 \mathrm{~cm}^{-1}$, atribuídas a modos de simetria $\mathrm{T}_{1 \mathrm{u}}$. Por fim, o complexo, $\left[\mathrm{Ni}\left(\mathrm{NH}_{3}\right)_{6}\right]^{2+}$ apresenta duas bandas referentes a modos de simetria $\mathrm{T}_{1 \mathrm{u}}$, e nos espectros vibracionais 
Tabela 3. Atribuição dos números de onda das bandas dos espectros vibracionais, infravermelho, das moléculas $\left[\mathrm{Ni}\left(\mathrm{NH}_{3}\right)_{6}\right]\left(\mathrm{ClO}_{4}\right)_{2},\left[\mathrm{Ni}_{(}\left(\mathrm{NH}_{3}\right)_{6}\right]\left(\mathrm{BF}_{4}\right)_{2}$ e $\left[\mathrm{Ni}\left(\mathrm{NH}_{3}\right)_{6}\right]$ $\left(\mathrm{PF}_{6}\right)_{2}$, e comparação com valores da literatura ${ }^{17}$

\begin{tabular}{|c|c|c|c|c|c|c|c|c|c|c|c|}
\hline \multicolumn{4}{|c|}{$\left[\mathrm{Ni}\left(\mathrm{NH}_{3}\right)_{6}\right]\left(\mathrm{ClO}_{4}\right)_{2}$} & \multicolumn{4}{|c|}{$\left[\mathrm{Ni}\left(\mathrm{NH}_{3}\right)_{6}\right]\left(\mathrm{BF}_{4}\right)_{2}$} & \multicolumn{4}{|c|}{$\left[\mathrm{Ni}\left(\mathrm{NH}_{3}\right)_{6}\right]\left(\mathrm{PF}_{6}\right)_{2}$} \\
\hline Molécula & Vibração & $\mathrm{cm}^{-1}$ & ${ }^{17}\left(\mathrm{~cm}^{-1}\right)$ & Molécula & Vibração & $\mathrm{cm}^{-1}$ & ${ }^{17}\left(\mathrm{~cm}^{-1}\right)$ & Molécula & Vibração & $\mathrm{cm}^{-1}$ & ${ }^{17}\left(\mathrm{~cm}^{-1}\right)$ \\
\hline $\mathrm{ClO}_{4}^{-}$ & $\mathrm{T}_{2}$ & 623 & 626 & $\mathrm{BF}_{4}^{-}$ & $\mathrm{T}_{2}$ & - & 548,6 & $\mathrm{PF}_{6}^{-}$ & $\mathrm{T}_{1 \mathrm{u}}$ & 555 & 560 \\
\hline$\left[\mathrm{Ni}\left(\mathrm{NH}_{3}\right)_{6}\right]^{2+}$ & $\mathrm{T}_{1 \mathrm{u}}$ & 650 & 672 & {$\left[\mathrm{Ni}\left(\mathrm{NH}_{3}\right)_{6}\right]^{2+}$} & $\mathrm{T}_{1 \mathrm{u}}$ & 653 & 676 & {$\left[\mathrm{Ni}\left(\mathrm{NH}_{3}\right)_{6}\right]^{2+}$} & $\mathrm{T}_{1 \mathrm{u}}$ & 647 & 654 \\
\hline $\mathrm{ClO}_{4}^{-}$ & $\mathrm{T}_{2}$ & 1076 & 1080 & $\mathrm{BF}_{4}^{-}$ & $\mathrm{T}_{2}$ & 1037 & 1060 & $\mathrm{PF}_{6}^{-}$ & $\mathrm{T}_{1 \mathrm{u}}$ & 825 & 830 \\
\hline$\left[\mathrm{Ni}\left(\mathrm{NH}_{3}\right)_{6}\right]^{2+}$ & $\mathrm{T}_{1 \mathrm{u}}$ & 1230 & 1240 & {$\left[\mathrm{Ni}\left(\mathrm{NH}_{3}\right)_{6}\right]^{2+}$} & $\mathrm{T}_{1 \mathrm{u}}$ & 1230 & 1238 & {$\left[\mathrm{Ni}\left(\mathrm{NH}_{3}\right)_{6}\right]^{2+}$} & $\mathrm{T}_{1 \mathrm{u}}$ & 1236 & 1240 \\
\hline $\mathrm{NH}_{3}$ & $\mathrm{E}$ & 1614 & 1622 & $\mathrm{NH}_{3}$ & $\mathrm{E}$ & 1621 & 1623 & $\mathrm{NH}_{3}$ & $\mathrm{E}$ & 1622 & 1628 \\
\hline $\mathrm{NH}_{3}$ & $\mathrm{~A}_{1}$ & 3297 & 3307 & $\mathrm{NH}_{3}$ & $\mathrm{~A}_{1}$ & 3311 & 3357 & $\mathrm{NH}_{3}$ & $\mathrm{~A}_{1}$ & 3315 & 3320 \\
\hline $\mathrm{NH}_{3}$ & $\mathrm{E}$ & 3380 & 3401 & $\mathrm{NH}_{3}$ & $\mathrm{E}$ & 3394 & 3412 & $\mathrm{NH}_{3}$ & $\mathrm{E}$ & 3401 & 3410 \\
\hline
\end{tabular}

Tabela 4. Atribuição dos números de onda das bandas dos espectros vibracionais, Raman, das moléculas $\left[\mathrm{Ni}\left(\mathrm{NH}_{3}\right)_{6}\right]\left(\mathrm{ClO}_{4}\right)_{2},\left[\mathrm{Ni}_{(}\left(\mathrm{NH}_{3}\right)_{6}\right]\left(\mathrm{BF}_{4}\right)_{2} \mathrm{e}\left[\mathrm{Ni}_{(}\left(\mathrm{NH}_{3}\right)_{6}\right]$ $\left(\mathrm{PF}_{6}\right)_{2}$, e comparação com valores da literatura ${ }^{17}$

\begin{tabular}{|c|c|c|c|c|c|c|c|c|c|c|c|}
\hline \multicolumn{4}{|c|}{$\left[\mathrm{Ni}\left(\mathrm{NH}_{3}\right)_{6}\right]\left(\mathrm{ClO}_{4}\right)_{2}$} & \multicolumn{4}{|c|}{$\left[\mathrm{Ni}\left(\mathrm{NH}_{3}\right)_{6}\right]\left(\mathrm{BF}_{4}\right)_{2}$} & \multicolumn{4}{|c|}{$\left[\mathrm{Ni}\left(\mathrm{NH}_{3}\right)_{6}\right]\left(\mathrm{PF}_{6}\right)_{2}$} \\
\hline Molécula & Vibração & $\mathrm{cm}^{-1}$ & ${ }^{17}\left(\mathrm{~cm}^{-1}\right)$ & Molécula & Vibração & $\mathrm{cm}^{-1}$ & ${ }^{17}\left(\mathrm{~cm}^{-1}\right)$ & Molécula & Vibração & $\mathrm{cm}^{-1}$ & ${ }^{17}\left(\mathrm{~cm}^{-1}\right)$ \\
\hline$\left[\mathrm{Ni}\left(\mathrm{NH}_{3}\right)_{6}\right]^{2+}$ & $\mathrm{T}_{2 \mathrm{~g}}$ & 234 & 241 & {$\left[\mathrm{Ni}\left(\mathrm{NH}_{3}\right)_{6}\right]^{2+}$} & $\mathrm{T}_{2 \mathrm{~g}}$ & 231 & 232 & {$\left[\mathrm{Ni}\left(\mathrm{NH}_{3}\right)_{6}\right]^{2+}$} & $\mathrm{T}_{2 \mathrm{~g}}$ & 224 & 229 \\
\hline$\left[\mathrm{Ni}\left(\mathrm{NH}_{3}\right)_{6}\right]^{2+}$ & $A_{1 g}$ & 360 & 363 & {$\left[\mathrm{Ni}\left(\mathrm{NH}_{3}\right)_{6}\right]^{2+}$} & $\mathrm{A}_{1 \mathrm{~g}}$ & 362 & 360 & {$\left[\mathrm{Ni}\left(\mathrm{NH}_{3}\right)_{6}\right]^{2+}$} & $A_{1 g}$ & 356 & 361 \\
\hline $\mathrm{ClO}_{4}^{-}$ & $\mathrm{E}$ & 462 & 465 & $\mathrm{BF}_{4}^{-}$ & $\mathrm{E}$ & 360 & 360 & $\mathrm{PF}_{6}^{-}$ & $\mathrm{T}_{2 \mathrm{~g}}$ & 470 & 470 \\
\hline $\mathrm{ClO}_{4}^{-}$ & $\mathrm{T}_{2}$ & 623 & 630 & $\mathrm{BF}_{4}^{-}$ & $\mathrm{T}_{2}$ & 524 & 525 & $\mathrm{PF}_{6}^{-}$ & $\mathrm{E}_{\mathrm{g}}$ & 580 & 585 \\
\hline $\mathrm{ClO}_{4}^{-}$ & $\mathrm{A}_{1}$ & 916 & 920 & $\mathrm{BF}_{4}^{-}$ & $\mathrm{A}_{1}$ & 770 & 770 & $\mathrm{PF}_{6}^{-}$ & $\mathrm{A}_{1 \mathrm{~g}}$ & 746 & 750 \\
\hline $\mathrm{ClO}_{4}^{-}$ & $\mathrm{T}_{2}$ & 937 & 940 & {$\left[\mathrm{Ni}\left(\mathrm{NH}_{3}\right)_{6}\right]^{2+}$} & $\mathrm{T}_{2 \mathrm{~g}}$ & 1045 & 1100 & {$\left[\mathrm{Ni}\left(\mathrm{NH}_{3}\right)_{6}\right]^{2+}$} & $\mathrm{T}_{2 \mathrm{~g}}$ & - & 1101 \\
\hline$\left[\mathrm{Ni}\left(\mathrm{NH}_{3}\right)_{6}\right]^{2+}$ & $\mathrm{T}_{2 \mathrm{~g}}$ & 1093 & 1102 & {$\left[\mathrm{Ni}\left(\mathrm{NH}_{3}\right)_{6}\right]^{2+}$} & $\mathrm{E}_{\mathrm{g}}$ & 1202 & 1220 & {$\left[\mathrm{Ni}\left(\mathrm{NH}_{3}\right)_{6}\right]^{2+}$} & $\mathrm{E}_{\mathrm{g}}$ & 1217 & 1222 \\
\hline$\left[\mathrm{Ni}\left(\mathrm{NH}_{3}\right)_{6}\right]^{2+}$ & $\mathrm{E}_{\mathrm{g}}$ & 1214 & 1215 & $\mathrm{NH}_{3}$ & $\mathrm{E}$ & 1621 & 1625 & $\mathrm{NH}_{3}$ & $\mathrm{E}$ & 1621 & 1620 \\
\hline $\mathrm{NH}_{3}$ & E & 1616 & 1615 & $\mathrm{NH}_{3}$ & $\mathrm{~A}_{1}$ & 3313 & 3320 & $\mathrm{NH}_{3}$ & $\mathrm{~A}_{1}$ & 3316 & 3320 \\
\hline $\mathrm{NH}_{3}$ & $\mathrm{~A}_{1}$ & 3301 & 3303 & $\mathrm{NH}_{3}$ & $\mathrm{E}$ & 3400 & 3400 & $\mathrm{NH}_{3}$ & $\mathrm{E}$ & 3403 & 3405 \\
\hline $\mathrm{NH}_{3}$ & $\mathrm{E}$ & 3385 & 3390 & & & & & & & & \\
\hline
\end{tabular}

obtidos para os complexos $\left[\mathrm{Ni}\left(\mathrm{NH}_{3}\right)_{6}\right]\left(\mathrm{ClO}_{4}\right)_{2},\left[\mathrm{Ni}\left(\mathrm{NH}_{3}\right)_{6}\right]\left(\mathrm{BF}_{4}\right)_{2} \mathrm{e}$ $\left[\mathrm{Ni}\left(\mathrm{NH}_{3}\right)_{6}\right]\left(\mathrm{PF}_{6}\right)_{2}$ as bandas aparecem em cerca de 650 e $1230 \mathrm{~cm}^{-1}$.

$\mathrm{O}$ mesmo tratamento pode ser feito para os modos ativos no Raman (Tabela 4). No caso do ligante $\mathrm{NH}_{3}$ duas bandas, em cerca de 1600 e $3400 \mathrm{~cm}^{-1}$, podem ser atribuídas aos modos vibracionais degenerados E, o primeiro referente a uma deformação angular e o segundo a um estiramento assimétrico. Uma banda em cerca de 3200 $\mathrm{cm}^{-1}$ é atribuída ao modo E e uma em $3300 \mathrm{~cm}^{-1}$ é atribuída ao modo

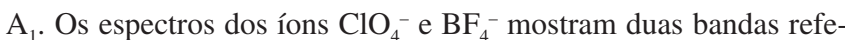
rentes ao modo $\mathrm{T}_{2}$, em 626 e $1100 \mathrm{~cm}^{-1}$, no caso do $\mathrm{ClO}_{4}^{-}$e somente uma banda em $525 \mathrm{~cm}^{-1}$, no caso do $\mathrm{BF}_{4}^{-}$, atribuídas a estiramentos. Os espectros mostram também uma banda referente ao modo $\mathrm{E}$ em

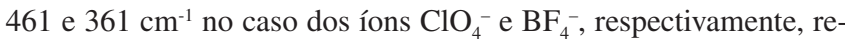
ferentes a deformações angulares. As bandas atribuídas aos modos $\mathrm{A}_{1}$ são observadas em 917 e $933 \mathrm{~cm}^{-1}$, no caso do $\mathrm{ClO}_{4}^{-}$, e somente uma banda em $771 \mathrm{~cm}^{-1}$, no caso do $\mathrm{BF}_{4}^{-}$, referentes a estiramentos axiais. $\mathrm{O}$ íon $\mathrm{PF}_{6}^{-}$apresenta uma banda em $470 \mathrm{~cm}^{-1}$ atribuída ao modo vibracional $\mathrm{T}_{2 \mathrm{~g}}$, uma banda em $580 \mathrm{~cm}^{-1}$ atribuída ao modo $\mathrm{E}_{\mathrm{g}}$, e uma banda em $746 \mathrm{~cm}^{-1}$ atribuída ao modo $\mathrm{A}_{1 \mathrm{~g}}$.

O complexo $\left[\mathrm{Ni}\left(\mathrm{NH}_{3}\right)_{6}\right]^{2+}$ apresenta uma banda referente ao modo $A_{1 g}$ nos três espectros obtidos e uma banda referente ao modo $E_{g}$, que aparece nos espectros dos complexos $\left[\mathrm{Ni}\left(\mathrm{NH}_{3}\right)_{6}\right]\left(\mathrm{ClO}_{4}\right)_{2} \mathrm{e}\left[\mathrm{Ni}\left(\mathrm{NH}_{3}\right)_{6}\right]$ $\left(\mathrm{BF}_{4}\right)_{2}$ em cerca de 1100 e $1200 \mathrm{~cm}^{-1}$.

Em um dos oferecimentos da disciplina, os alunos responderam a um questionário de avaliação da disciplina. Nesse oferecimento, os alunos não realizaram o experimento, mas apenas utilizaram os dados que seriam obtidos como exercício. Não é possível fazer uma análise estatística, uma vez que foram apenas 9 questionários respondidos e o questionário não foi aplicado em todos os oferecimentos da disciplina. Entretanto, alguns pontos levantados pelos alunos são bastante interessantes. Os alunos mostraram-se bastante satisfeitos em relação às três dimensões abordadas e a maior parte dos pontos levantados foram avaliados como ótimo ou bom. Entretanto, no que concerne à autoavaliação, muitos consideraram sua capacidade para acompanhar a disciplina como regular. Por outro lado, nos itens que avaliavam os experimentos, a maior parte assinalou "nenhuma das respostas", como esperado, mas um aluno sugeriu que a disciplina contasse com uma parte prática e outro apontou que a utilização de resultados de experimentos, mesmo que não tendo sido obtidos por eles, foi bastante ilustrativa.

Por outro lado, em outro oferecimento da disciplina, os alunos foram organizados em grupos para realizar o experimento e depois discutir e propor a atribuição do espectro em função da tabela de caracteres. As discussões foram bastante frutíferas e essa atividade parece ter contribuído para uma aprendizagem mais significativa, pois apresentaram um melhor desempenho nas avaliações em relação aos alunos dos outros oferecimentos da disciplina.

Adicionalmente, seria possível, também, realizar cálculos de química quântica (no caso de alunos que já tenham estudado os princípios da mecânica quântica) com o intuito de proporcionar a visualização dos modos vibracionais.

$\mathrm{Na}$ UFABC, as disciplinas Estrutura da Matéria (com $36 \mathrm{~h}$ de aulas teóricas, cursada no $1^{\circ}$ quadrimestre) e Física Quântica (também com $36 \mathrm{~h}$ de aula, cursada no $5^{\circ}$ quadrimestre) são obrigatórias para o curso de ingresso, o Bacharelado em Ciência e Tecnologia e, consequentemente, para o Bacharelado em Química. No Bacharelado 
em Química, os alunos ainda cursam a disciplina Mecânica Quântica, de $72 \mathrm{~h}$, no $8^{\circ}$ quadrimestre.

A realização de cálculos de química quântica durante a disciplina Ligações Químicas, portanto, pode trazer também como benefício um incentivo ao estudo da mecânica quântica, mostrando um lado mais "aplicado" dessa área do conhecimento. A importância da utilização de projetos desenvolvidos com apoio de computadores e softwares de mecânica quântica foi abordada anteriormente. ${ }^{19}$

$\mathrm{O}$ estudo poderia, inclusive, ser aprofundado para entender as bases da mecânica quântica em função da teoria de grupos. O livro de Fazzio e Watari ${ }^{20}$ pode ser utilizado com esse fim e possui uma linguagem apropriada ao nível de graduação.

\section{CONCLUSÃO}

Foi apresentado um experimento utilizado na disciplina Ligações Químicas, dos Bacharelados em Ciência e Tecnologia e em Química, da Universidade Federal do ABC, com o intuito de facilitar e motivar o ensino e a aprendizagem de teoria de grupos. Apresentamos alguns aspectos fundamentais dessa teoria que esperamos possam servir para indicar os caminhos para o estudo e aprofundamento no tema.

A aprendizagem dos alunos durante o oferecimento em que o experimento foi utilizado mostrou-se mais significativa, pois houve um aumento no nível das discussões entre os grupos de alunos e no desempenho nas avaliações. Neste trabalho também é proposta uma abordagem multidisciplinar ao incluir a utilização de ferramentas de química quântica. Cabe, ressaltar, entretanto, que uma discussão mais completa, levando em conta os grupos de simetria cristalina, se faz necessária e será alvo de trabalhos futuros.

\section{MATERIAL SUPLEMENTAR}

Está disponível em http://quimicanova.sbq.org.br, na forma de arquivo PDF e com acesso livre.

\section{AGRADECIMENTOS}

À FAPESP (Proc. No. 2008/53576-9; 2007/05370-0; 2010/098917), CNPq (Proc. No. 474871/2010-0) e ao INCT de Bioanalítica (CNPq, Proc. No. 573672/2008-3 e FAPESP, Proc. No. 08/57805-2) pelo suporte financeiro.

\section{REFERÊNCIAS}

1. Marcolin, N.; Pesquisa FAPESP 2011, 8.

2. Bishop, D. M.; Group Theory and Chemistry, Dover Publications, Inc.: New York, 1993.

3. Cotton, F. A.; Chemical Applications of Group Theory, $3^{\text {rd }}$ ed., Wiley: New Work, 1990.

4. Hayers, W.; Loudon, R.; Scattering of Ligh By Crystals, Dover Publications, Inc.: New York, 1978.

5. Oliveira, G. M.; Simetria de Moléculas e Cristais: Fundamentos de Espectroscopia Vibracional, Bookman: Porto Alegre, 2009.

6. Tsukerblat, B. S.; Group Theory in Chemistry and Spectroscopy: a Simple Guide to Advanced usage, Dover Publications: Mineola, 2006.

7. Harris, D. C.; Bertolucci, A. D.; Symmetry and Spectroscopy: an Introduction to Vibrational and Electronic Spectroscopy, Dover Publications: New York, 1989.

8. Sala, O.; Espectroscopia Raman e no Infravermelho, $2^{\mathrm{a}}$ ed., Ed. da UNESP: São Paulo, 2008.

9. Hollas, J. M.; Modern Spectroscopy, Wiley inc.: New Jersey, 2004.

10. Sala, O.; Quim. Nova 2008, 31, 914.

11. Woolins, J. D.; Inorganic Experiments, $2^{\text {nd }}$ ed., Wiley - VHC: Weinheim, 2003.

12. White, J. E.; J. Chem. Educ. 1967, 44, 128; Burrows, E. L.; Clark, M. J.; J. Chem. Educ. 1974, 51, 87; Thomas, C. H.; J. Chem. Educ. 1974, $51,91$.

13. Sala, O.; Quim. Nova 2007, 30, 1773; Sala, O.; Quim. Nova 2007, 30, 2057; de Oliveira, L. F. C.; Quim. Nova na Escola 2001, n 4, 24; de Almeida, W. B.; dos Santos, H. F.; Quim. Nova na Escola 2001, n 4, 6; Azevedo, E. R. de; Nunes, L. A. O.; Quim. Nova 2008, 31, 2199.

14. Gushikem, Y.; Quim. Nova 2005, 28, 153.

15. Huheey, J. E.; Keiter, E. A.; Keiter, R. L.; Inorganic Chemistry: Principles of Structure and Reactivity, th $^{\text {th }}$ ed., Harper Collins College Publishers: New York, 1993.

16. Woolins, J. D.; Inorganic Experiments, $2^{\text {nd }}$ ed., Wiley - VHC: Weinheim, 2003.

17. Davidson, G.; Introductory Group Theory for Chemists, Applied Science Publishers LTD: London, 1971.

18. Kulczycki, A.; J. Phys. C: Solid State Phys. 1981, 14, 2433.

19. Arroio, A.; Honório, K. M.; Weber, K. C.; Homem-de-Mello, P.; Silva, A. B. F.; Quim. Nova 2005, 28, 360.

20. Fazzio, A.; Watari, K.; Introdução à Teoria de Grupos com Aplicações em Moléculas e Sólidos, Ed. da UFSM: Santa Maria, 1998. 